\title{
LA CONSTRUCCIÓN Y JUSTIFICACIÓN DE IDEAS MATEMÁTICAS GENERALES POR UN NIÑO DE 5 AÑOS: EL ROL DE LAS REPRESENTACIONES MANIPULATIVAS
}

\section{GENERALIZING AND JUSTIFYING MATHEMATICAL IDEAS BY A 5-YEAR- OLD CHILD: THE ROLE OF MANIPULATIVE REPRESENTATIONS}

\author{
Eder Pinto \\ Universidad del Desarrollo - Chile \\ epinto@udd.cl
}

\section{Resumen}

Diversos reportes de investigación han mostrado cómo niños ${ }^{1}$ de las primeras edades construyen ideas matemáticas generales, aspecto central del pensamiento algebraico. Sin embargo, los estudios que abordan cómo estudiantes de estas edades justifican esas ideas generales son escasos y esto constituye la originalidad y contribución de esta investigación. Considerando el rol predominante de las representaciones manipulativas en las experiencias matemáticas de niños de las primeras edades, la pregunta de investigación que busco responder es: ¿cómo las representaciones manipulativas apoyan la construcción y justificación de ideas matemáticas generales al resolver problemas que tienen un carácter algebraico? Para aproximarme a dicha pregunta, realicé una entrevista individual semiestructurada a un niño de 5 años, en la cual le presenté dos problemas diferentes que buscaban que el participante atendiera a la relación entre una determinada cantidad de perros y la cantidad de colas/ojos respectiva. Los resultados muestran que a pesar de no emplear y/o referirse al material manipulativo para construir reglas generales y/o justificar, el participante encontró relaciones generales entre las variables involucradas en los problemas. Asimismo, sus justificaciones se basan en las reglas generales que él construyó. Finalmente, el participante extiende dichas reglas generales hasta el punto de interactuar con cantidades indeterminadas.

Palabras claves: pensamiento algebraico, generalización, justificación, representaciones manipulativas

\begin{abstract}
Different studies have shown how early childhood students generalize mathematical relationships, a crucial aspect of early algebraic thinking. However, studies that focus on how these students justify these general ideas are scarce and this constitutes the originality and contribution of this research. Considering the predominant role of manipulative representations in the mathematical

${ }^{1}$ En este documento se utilizan de manera inclusiva términos como "los estudiantes", "los niños", "los investigadores" para aludir a hombres y mujeres. Esta opción obedece a que no existe acuerdo universal respecto de cómo nombrar conjuntamente a ambos sexos en el idioma español, salvo usando "o/a", "los/las" y otras similares, y ese tipo de fórmulas supone una saturación gráfica que puede dificultar la comprensión de la lectura.
\end{abstract}


experiences in Early Childhood Education, the research question that addresses this work is: How do manipulative representations support the construction and justification of general mathematical ideas child when solving problems that have an algebraic features? To answer this question, I conducted a semi-structured individual interview with 5-years-old children, in which I posed two different problems that sought for the participant to address the relationship between a certain number of dogs and the respective number of tails / eyes. The results show that despite not using and / or referring to manipulative material to construct rules and/or general justifications, the participant finded general relationships between the variables involved in problems. Likewise, his justifications are based on the general rules that he constructed. Finally, the participant extended these general rules to the point of interacting with indeterminate quantities.

Keywords: early algebraic thinking, generalization, justification, manipulative representations.

\section{INTRODUCCIÓN}

Actualmente existe suficiente evidencia científica para demostrar que niños comienzan a desarrollar su pensamiento matemático antes de iniciar la educación básica (6-7 años de edad) (e.g., BAROODY, 2004; CASTRO; CASTRO, 2016; FUSON, 2004; PIAGET; INHELDER, 1967; SARAMA; CLEMENTS, 2009). Más específicamente, diferentes autores han reconocido que antes de llegar al colegio, los estudiantes están naturalmente predispuestos a percibir regularidades y encontrar relaciones matemáticas generales (BASTABLE; SCHIFTER, 2008; MASON, 1996), aún cuando estos no tienen todas las representaciones para expresar dichas ideas generales. El pensamiento algebraico aprovecha la capacidad natural que tienen niños para capturar relaciones matemáticas generales con fines didácticos (MASON, 1996) y es el contexto general de este artículo.

El pensamiento algebraico es un tipo particular de pensamiento matemático, el cual involucra atender a las relaciones entre cantidades variables e indeterminadas (no específicas), reconocer estructuras, estudiar cambios, generalizar, resolver problemas, modelizar, justificar, probar y predecir. Este tipo de pensamiento puede tomar lugar en ausencia de representaciones simbólicas como letras y números; permite que niños puedan usar representaciones tan variadas como materiales manipulativos, dibujos, lenguaje natural, números, entre otros (CARRAHER; SCHLIEMANN, 2010). El desarrollo y fomento de este tipo de pensamiento en la Educación Parvularia ${ }^{2}$ tiene sentido dado que la introducción de conceptos algebraicos está presente en los currículos matemáticos de diferentes países. En Chile, por ejemplo, las Bases Curriculares para la

${ }^{2}$ De aquí en adelante usaré este término para referirme al ciclo educativo que atiende a estudiantes de 0-6 años. 
Educación Parvularia (SUBSECRETARÍA DE EDUCACIÓN PARVULARIA, 2018) incorporan la idea de patrones desde los 2 años; un tema tradicional del álgebra. Específicamente, se espera que niños de 2-4 años logren "reproducir patrones sonoros, visuales, gestuales, corporales y otros, de dos o tres elementos" (p. 98). Este tipo de actividades requiere que los profesores diseñen actividades que vayan más allá de encontrar el siguiente término en una secuencia dada. Por tanto, se hace necesario brindar herramientas útiles que favorezcan la promoción y desarrollo del pensamiento algebraico en niños de estas edades.

De manera general, en este artículo describiré cómo un niño de 5 años construye relaciones matemáticas generales (generaliza) y justifica sus ideas. Dos razones principales dirigen este artículo. En primer lugar, y desde la perspectiva del pensamiento algebraico, son escasos los estudios que abordan detalladamente cómo los niños en edad preescolar justifican las ideas generales que van construyendo. Diferentes autores enfatizan la idea que las escuelas deben fomentar en estudiantes de 4 hasta 14 años la capacidad de construir justificaciones matemáticas para convencerse a sí mismos y a otros del porqué una afirmación general es verdadera (LANNIN; ELLIS; ELLIOT, 2011). La justificación en cualquier forma, y tal como lo señalan Blanton e Kaput (2000), es parte importante del pensamiento algebraico, ya que induce un hábito mental por el cual uno naturalmente cuestiona y conjetura para establecer una generalización. Por otra parte, existen diferentes investigaciones que muestran cómo niños de estas edades construyen relaciones generales al tratar con diferentes contenidos de carácter algebraico (BLANTON; OTÁLORA; BRIZUELA; GARDINER; SAWREY; GIBBINS; KIM, 2018; BLANTON; KAPUT, 2004; CASTRO; CAÑADAS; MOLINA, 2017; RAMIREZ; BRIZUELA; BLANTON, 2020). Es así como se hace necesario profundizar en la manera que se relacionan las actividades de generalizar y justificar; componentes esenciales de la actividad algebraica (ELLIS, 2007).

En segundo lugar, me interesa indagar cómo las representaciones manipulativas las cuales son ampliamente utilizadas en las experiencias matemáticas con estudiantes de 0-6 años-interactúan con las generalizaciones y justificaciones que niños de estas edades construyen. Tal como señalaba anteriormente, el desarrollo del pensamiento algebraico va más allá del uso de letras y símbolos; permite el empleo de variadas representaciones con 
la intención de que los estudiantes vayan centrando su atención en relaciones matemáticas generales más que en detalles.

En un reciente estudio, Castro et al. (2017) muestran evidencias de cómo un grupo de 12 niños de 5-6 años interactúan con contenidos algebraicos durante algunas sesiones de clases. Las investigadoras presentaron diferentes problemas orales, relacionados con una cuidadora de perros y acompañados de un material manipulativo que colocaron en la pizarra (figuras de perros y platos). Uno de los problemas involucraba la relación entre una cantidad de perros y platos: "Para un perro ponemos dos platos. Para dos perros ponemos cuatro platos. Para tres perros ponemos seis platos. Y para cuatro perros, ¿cuántos platos son?” (p. 6). Así, los niños respondieron a diferentes preguntas que involucraban dicha relación, atendiendo a las variables insertas en el problema. Dentro de los resultados presentados, las autoras señalan que los estudiantes se apoyaban en el material concreto para contar y responder a las primeras preguntas (que involucran casos numéricos pequeños). Luego, cuando el ámbito numérico se hacía más grande, ellos dejaban de contar, "no solo porque ese número 'elevado' de perros no esté presente en la pizarra, sino porque no les es necesario ya que han percibido el patrón" ( $p$. 8). Este trabajo ha servido como inspiración para este artículo y abre una interesante línea: indagar con mayor profundidad en cómo niños de las mismas edades generalizan y justifican dichas ideas, usando representaciones manipulativas. Esta indagación la realizaré mediante una entrevista, la cual me permitirá comprender como un niño de 5 años va interactuando con representaciones manipulativas para construir y justificar relaciones matemáticas generales.

De manera general, el enfoque teórico de este estudio sigue las ideas de Kaput (2008), el cual resalta que el pensamiento algebraico está compuesto por un proceso de simbolización que tiene como propósito la generalización y el razonamiento con generalizaciones. Específicamente, me centro en una aproximación funcional al pensamiento algebraico (CARRAHER; SCHLIEMANN, 2007), el cual es considerado un vehículo para introducir álgebra en los primeros cursos. En este tipo de enfoque, la función es el contenido matemático clave ya que favorece que los estudiantes: (a) atiendan a las relaciones y estructuras que subyacen en problemas que involucran la variación entre cantidades, en vez de centrarse en cálculos aritméticos aislados; (b) 
unifiquen ideas y procedimientos que pueden no estar relacionados; (c) mejoren su capacidad para generalizar; (d) construyan conceptos matemáticos: y (e) relacionen e integren diferentes ramas de las matemáticas, como la aritmética, álgebra y geometría (BLANTON, 2008; CARRAHER; SCHLIEMANN, 2015).

En este artículo describo cómo un niño de 5 años construye relaciones matemáticas generales y justifica la veracidad de dichas relaciones. Considerando el uso de la representación manipulativa, la pregunta de investigación que dirige el trabajo es: ¿cómo las representaciones manipulativas apoyan la construcción y justificación de ideas matemáticas generales al resolver problemas que tienen un carácter algebraico?

\section{ENFOQUE FUNCIONAL AL PENSAMIENTO ALGEBRAICO}

Existen tres aproximaciones al pensamiento algebraico: aritmética generalizada; equivalencia, expresiones, ecuaciones e inecuaciones; y enfoque funcional. Cada una de estas aproximaciones resaltan diferentes contenidos algebraicos. En concreto, la aproximación funcional al pensamiento algebraico "involucra la generalización de relaciones que covarían, las cuales pueden ser generalizadas o no, y son expresadas mediante diferentes representaciones" (PINTO, 2019, p. 106). Por ejemplo, un problema bajo este enfoque es el que presentan Torres, Cañadas, Moreno y Gómez (2021) a niños de 7-8 años de edad y lo muestro en la Figura 1.

Iron Man y el Capitán América cumplen años el mismo día. Cuando Iron Man cumplió 5 años, el Capitán América cumplió 9. Cuando Iron Man cumplió 7 años, el Capitán América cumplió 11 .

- Dime una edad para Iron Man. Si cumple esos años, ¿cuántos años cumple el Capitán América?

- ¿Cómo le explicarías a un amigo que ha de hacer para conocer la edad del Capitán

Figura 1. Problema de las edades de los superhéroes

Si bien la función no es un contenido matemático que se encuentra en los currículos de los primeros cursos, diferentes autores (e.g., CAÑADAS; MOLINA, 2016; CARRAHER; SCHLIEMANN, 2007) resaltan que este concepto es un contexto para fomentar el pensamiento algebraico y que puede ser empleado en problemas contextualizados (ver Figura 1) o en problema de patrones geométricos. Tal como lo presento en la Figura 1, el problema de las edades establece la relación entre dos variables: la edad de Iron Man (variable independiente) y la edad del capitán América 
(variable dependiente). Tal como se muestra en este problema, la función del problema $(y=x+4)$ está implícita en el problema. La primera pregunta atiende a la relación entre las variables para un caso particular. En cambio, la segunda pregunta aborda la relación general.

Desde el enfoque funcional al álgebra escolar, generalizar implica atender, percibir y expresar cómo una cantidad varía con respecto a la otra en general (BLANTON, 2008, 2017), identificando un patrón que sea válido para más casos, a partir de la regularidad observada. Por tanto, la generalización se da al establecer y analizar las relaciones entre variables (SMITH, 2003) y los problemas que involucran funciones se transforman en un escenario óptimo para que los estudiantes identifiquen patrones y generalizaciones (WARREN, 2005).

Si bien la justificación no es en sí misma algebraica, esta tiene un propósito algebraico cuando los estudiantes justifican afirmaciones generalizadas (BLANTON; BRIZUELA, STEPHENS, KNUTH; ISLER..., 2018). Desde esta perspectiva, la justificación proporciona una idea de las relaciones matemáticas que subyacen a la generalización, por tanto, la justificación permite comprender porqué dicha afirmación general es verdadera (RUSSELL; SCHIFTER; BASTABLE, 2011).

En las representaciones manipulativas o concretas — como las regletas Cuisenaire o bloques multibase - los elementos que las conforman "tienen poco significado per se, pero las relaciones y operaciones integradas se ajustan a muchas situaciones cotidianas" (Lesh et al., 1987, p. 33). Baroody (1990) señala que el uso de este tipo de representación no es garantía de una comprensión conceptual de los estudiantes, ni tampoco lleva automáticamente a los estudiantes a generalizar (Cai y Moyer, 2008).

\section{METODOLOGÍA}

La investigación que presento es cualitativa, dado que el interés está puesto en comprender y describir los fenómenos, "explorándolos desde la perspectiva de los participantes en un ambiente natural y en relación al contexto" (HERNÁNDEZ; FERNÁNDEZ; BAPTISTA, 2020, p. 364). Específicamente, presento un estudio que describe la forma en que un niño de 5 años emplea representaciones manipulativas para generalizar y justificar sus ideas matemáticas. Esta muestra ha sido intencional. Para obtener los datos, diseñé e implementé una entrevista individual semiestructurada. 
Pinto, E.

\section{Participante}

Escogí a un niño de 5 años y 4 meses, quien al momento de la entrevista estaba iniciando Kinder ${ }^{3}$. Lo seleccioné por su gusto por las matemáticas y por su capacidad para expresar ideas. Para proteger su identidad, llamaré $\mathrm{T}$ al participante de esta investigación. Durante el curso anterior (Pre-Kinder), T participó de sus clases de matemáticas manera online, dado el contexto sanitario producido por la pandemia SARSCoV-2. Durante dicho período académico, él participó de dos sesiones de matemáticas a la semana, de 45 minutos cada una. Dentro de sus conocimientos previos, $\mathrm{T}$ representa números y cantidades hasta el 20, de manera concreta, pictórica y simbólica. Emplea los números para ordenar, identificar, cuantificar y comparar hasta el 20, en situaciones cotidianas y juegos. En cuanto al trabajo con patrones, $\mathrm{T}$ reconoce patrones cualitativos de hasta tres elementos.

\section{Entrevista individual semiestructurada}

Diseñé una entrevista con la intención de profundizar en las ideas que tienen un niño de 5 años al construir relaciones matemáticas generales y cómo justifica la veracidad de dichas ideas generales. Para ello, mi interés está dado por la interacción y uso de representaciones manipulativas que presenté a $\mathrm{T}$.

La entrevista como instrumento para la recolección de información es "una herramienta flexible para la recopilación de datos, que permite utilizar canales multisensoriales: verbales, no verbales, vistos, hablados, escuchados $\mathrm{y}$, de hecho, con entrevistas en línea o escritas" (COHEN; MANION; MORRISON, 2018, p. 506). Específicamente, desarrollé una entrevista individual semiestructurada, en la cual el entrevistador es libre de modificar, de acuerdo con las respuestas de los estudiantes, la secuencia de las preguntas, las cuales se acomodan a las respuestas de los estudiantes y permiten obtener información más específica (GINSBURG, 1997). Entrevisté a T en dos momentos diferentes. Cada entrevista tuvo una duración de 10-15 minutos, las cuales fueron vídeo-grabadas.

Considerando el propósito de la investigación, adapté dos problema usados por

\footnotetext{
${ }^{3}$ En Chile, las Bases Curriculares para la Educación Parvularia (Subsecretaría de Educación Parvularia, 2018) distinguen tres niveles curriculares, los cuales abarcan tramos de dos años cada uno. El primer nivel (sala cuna) abarca a niños hasta los 2 años. El segundo nivel (medio) aborda desde los dos hasta los cuatros años. Finalmente, el tercer nivel (Transición) está dirigido para niños de hasta seis años de edad. Dentro de este último nivel, por lo general, se distinguen dos cursos: Pre-Kinder (4-5 años) y Kinder (5-6 años).
} 
diferentes colegas: (a) el problema de los perros y las colas; y (b) el problema de los perros y los ojos (BLANTON; KAPUT, 2004; CASTRO; CAÑADAS; MOLINA, 2017; MORALES; CAÑADAS; BRIZUELA; GÓMEZ, et al., 2018). Estos problemas lo seleccioné por: (a) brindar un contexto cercano para niños de 5 años; (b) ofrecer la oportunidad de que niños interactúen con diferentes tipos de funciones lineales; y (c) permitir establecer comparaciones con estudios previos. Los problemas los presenté de manera oral a $\mathrm{T}$ y los trabajamos en dos entrevistas de igual duración.

\section{Entrevista 1: El problema de los perros y las colas.}

En la primera entrevista presenté el problema de los perros y las colas, el cual permite identificar regularidades entre la cantidad de perros y la respectiva cantidad de colas (y viceversa). En la Figura 2 presento el problema empleado.

\section{(a) Problema de perros y colas (relación directa)}

Supongamos que estamos en un refugio para perros y quieres contar todas las colas de los perros que observaste.

- Si hay 1 perro, ¿cuántas colas hay en total?

- ¿Y en 2 perros?

- ¿Y en 3 perros?

- ¿Y en 8 perros?

- ¿Y en 5 perros?

- ¿Y en cien perros?

- ¿Y en un millón de perros?

- ¿Y son muchísimos perros, no sé cuántos, solo sé que muchísimos?

- ¿Ves alguna relación o algo que esté ocurriendo entre el número de perros y el número de colas? ¿Cómo lo sabes?

(b) Problema de perros y colas (relación inversa)

tu conoces cuántas colas hay en total y necesito que me digas cuántos perros hay.

- Si hemos contado 1 cola, ¿cuántos perros hay en total?

- ¿Y si contamos 2 colas?

- ¿Y 3 colas?

- ¿Y 8 colas?

- ¿Y 5 colas?

- ¿Y cien colas?

- ¿y un millón de colas?

- ¿Y son "muchísimas" colas, no sé cuántas, solo sé que "muchísimas"?

- ¿Ves alguna relación o algo que esté ocurriendo entre el número de perros y el número de colas? ¿Cómo lo sabes?

Figura 2. Problema de los perros y colas.

Este problema (ver Figura 2) lo presenté oralmente y lo acompañé de un material manipulativo: figuras de perritos. Utilicé este material para apoyar la comprensión del problema y para observar en qué medida este tipo de representación favorecería la 
construcción de ideas generales y su justificación. El problema lo organicé en dos momentos. En (a) presenté preguntas que abordan la relación directa (dado el valor de la variable independiente, determinar el valor de la variable dependiente) y la función involucrada es $y=x$. Por tanto, en (a) presenté preguntas que buscan determinar la cantidad de colas dada una cantidad de perros (e.g., si tenemos 5 perros, ¿cuántas colas hay en total?). En (b) las preguntas abordan la relación inversa (dado un número de colas, determinar la cantidad de perros). En ambas situaciones se presentan preguntas que involucran:

- cantidades cercanas no consecutivas $(1,2,3,8$ y 5);

- cantidades lejanas (“cien”, “un millón”);

- cantidades indeterminadas ("muchísimos", "muchísimas”); y

- la relación entre las cantidades involucradas (¿Ves alguna relación o algo que esté ocurriendo entre el número de perros y el número de colas? ¿Cómo lo sabes?)

Esta organización de preguntas sigue el modelo de razonamiento inductivo propuesto por Cañadas y Castro (2007). Adicionalmente, y para apoyar el proceso de generalización y justificación de $\mathrm{T}$, realicé diferentes tipos de preguntas, tal como aparece en la Tabla 1.

Tabla 1. Preguntas para apoyar procesos de generalización y justificación

\begin{tabular}{|c|c|}
\hline Foco & Preguntas \\
\hline $\begin{array}{l}\text { Explicar estrategias e } \\
\text { ideas, contrastar ideas }\end{array}$ & $\begin{array}{l}\text { - ¿En qué estás pensando para responder así? } \\
\text { - ¿Cómo lo estás pensando? } \\
\text { - ¿Lo podrías hacer de otra forma? } \\
\text { - ¿Podrías darme un ejemplo? } \\
\text { - Si un amigo te dice que XXXXX, ¿estarías de acuerdo con él? }\end{array}$ \\
\hline Describir conjeturas & $\begin{array}{l}\text { - ¿Te das cuento que algo siempre está ocurriendo? } \\
\text { - ¿Esto siempre será así? } \\
\text { - ¿Cómo convencerías a tu amigo que esto siempre es así? }\end{array}$ \\
\hline Justificar sus conjeturas & $\begin{array}{l}\text { - ¿Cómo sabes que esa idea es siempre verdadera? } \\
\text { - ¿Cómo sabes que tus respuestas son siempre correctas? } \\
\text { - ¿Cómo podrías convencer a un/a amigo/a de tus ideas? }\end{array}$ \\
\hline $\begin{array}{l}\text { Para garantizar una } \\
\text { justificación completa de } \\
\text { las soluciones de los } \\
\text { estudiantes: }\end{array}$ & $\begin{array}{l}\text { - ¿Qué hice? } \\
\text { - ¿Por qué lo hice? } \\
\text { - Si cambié de opinión, ¿por qué? } \\
\text { - Si no respondí, ¿por qué? }\end{array}$ \\
\hline
\end{tabular}


Las preguntas de la Tabla 1 han sido empleadas por investigaciones previas que tratan aspectos del pensamiento algebraico (AMIT; NERIA, 2008; BLANTON, 2008; BOALER; HUMPHREYS, 2005; RUSSELL ET AL., 2011).

\section{Entrevista 2: El problema de los perros y los ojos.}

En la segunda entrevista presenté a $\mathrm{T}$ un problema que involucra el número de perros y la cantidad de ojos total $(y=2 x)$. Este problema involucra la estructura multiplicativa y solo realicé preguntas que involucran la relación directa (dado un número de perros, determinar la cantidad de ojos). La Figura 3 presenta el problema presentado.

\section{Problema de perros y ojos (relación directa)}

Seguimos en el refugio de perros y estamos interesados en contar todos los ojos de los perros que vas observando.

- Si hay 1 perro, ¿cuántos ojos hay en total?

- ¿Y en 2 perros?

- ¿Y en 3 perros?

- ¿Y en 8 perros?

- ¿Y en 5 perros?

- ¿Y en 100 perros?

- ¿Y 1.000 .000 de perros?

- ¿Y son muchísimos perros, no sé cuántos, solo sé que muchísimos?

- ¿Ves alguna relación o algo que esté ocurriendo entre el número de perros y el número de colas?

- ¿Cómo describirías esa relación? ¿Cómo lo sabes?

Figura 3. Problema de los perros y los ojos.

Al igual que en la primera entrevista, este problema lo presenté de manera oral y con apoyo del material manipulativo (figuras de perros). La organización de las preguntas siguió la misma estructura anterior: (a) cantidades cercanas no consecutivas; (b) cantidades lejanas; (c) cantidades indeterminadas; y (d) la relación entre las cantidades involucradas.

\section{Análisis de datos}

La información recopilada para esta investigación es la video-grabación de las respuestas de T, así como la transcripción de sus respuestas. Concretamente, Analicé las respuestas orales de $\mathrm{T}$ al responder a los diferentes problemas planteados. Concretamente, identifiqué en qué momentos de la entrevista él usaba o hacía referencia al material manipulativo proporcionado. De manera simultánea, analicé los momentos en que T construía relaciones generales y justificaba sus ideas. 


\section{RESULTADOS}

En esta sección presento los resultados según el tipo de función involucrada en cada problema. Para ello, y considerando la pregunta de investigación (¿Cómo las representaciones manipulativas apoyan la construcción y justificación de ideas matemáticas generales al resolver problemas que tienen un carácter algebraico?), me centraré en describir los momentos en los cuales T usó o hizo referencia al material manipulativo proporcionado.

\section{El problema de los perros y las colas (relación directa, $y=x$ )}

Luego de presentar el contexto del problema y de realizar preguntas sobre la comprensión de este (¿sabes lo que es un refugio para perros? ¿qué podemos encontrar ahí? ¿cuántas colas tiene un perro?, ¿y ojos?), pregunté a T cuántas colas hay si tenemos 1, 2, 3, 8 y 5 perros. Para los dos primeros casos (1 y 2 perros), T dio su respuesta inmediatamente y para justificarla, se apoyó en la representación manipulativa. El siguiente extracto es un ejemplo de esta situación.

1. Entrevistador $(\mathrm{E})$ : $\quad$ Si yo tengo un perrito, ¿cuántas colas hay en total?

2. T: Una.

3. E: ¿Cómo lo sabes?

4. T: Porque tiene una (cola) (y apoya el dedo sobre la cola, ver Figura 4).

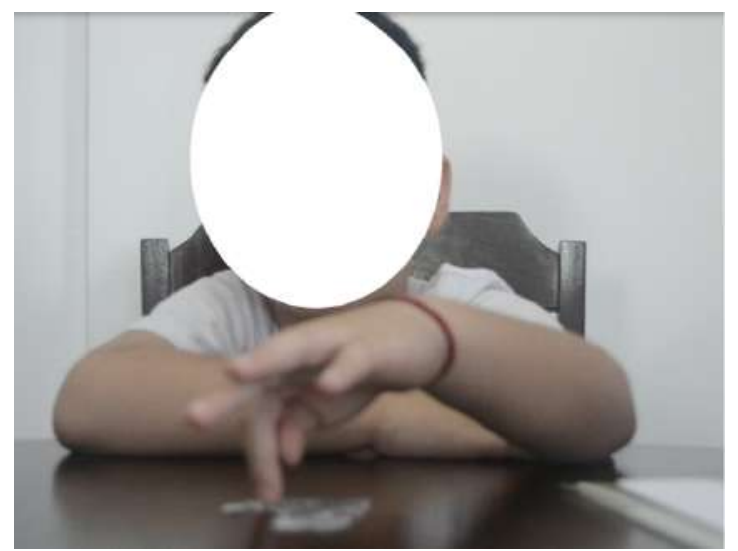

Figura 4. Señalando la cola de 1 perro

5. E:

6. T:

7. E:
Y si ahora hay dos perros en el refugio, ¿cuántas colas hay en total?

Dos.

¿Cómo lo sabes? 
8. T:

Porque hay dos perros (apunta a los perros, ver Figura 5).

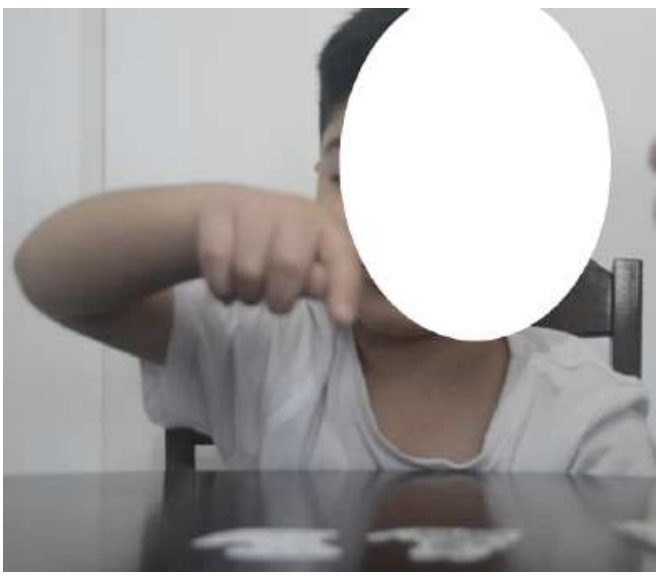

Figura 5. Señalando la cantidad de colas de 2 perros

Tal como se muestra, el uso de la representación manipulativa fue empleada por T para justificar que el número de colas era el que se observaba en las figuras. Luego, cuando pregunté por la cantidad de colas total para 3,8 y 5 perros, T prescinde de la representación manipulativa para responder y justificar su razonamiento. El siguiente extracto es un ejemplo de las respuestas de $\mathrm{T}$.

9. E:

Ahora vamos a pensar que hay ocho perritos,

10. T: ¿cuántas colas habrá en total?

Eh, ocho.

11. E: ¿Por qué?

12. T: Porque hay ocho perros.

Luego, y dado que $\mathrm{T}$ justifica siempre de la misma forma (porque hay $3 / 8 / 5$ perros), le pregunté por la regularidad que él observa en esta situación. El siguiente diálogo ilustra un ejemplo de esta situación.

13. E:

14. T:

15. E:

16. $\mathrm{T}$ :
¿Cómo lo haces para responder el número de colas?, ¿qué es lo que estás pensando siempre?

Porque tu lo decís con el número... Explícame mejor esa idea, ¿a qué te refieres? Es que tú decís el número y yo digo las colas con ese número. 
Tal como se muestra en el extracto anterior, $\mathrm{T}$ explica una regularidad que para él está ocurriendo siempre (ver líneas 14 y 16). Nuevamente, T no interactúa con el material manipulativo para responder. Posteriormente, y para profundizar en la idea "porque tu lo decís con el número" (ver línea 14), le pregunté a T por la cantidad de colas para cien y un millón de perros (cantidades lejanas).

17. $\mathrm{E}: \quad$ Ahora te voy a decir un número muy grande...

18. T: ¿Cómo el cien?

19. E: Vale, como el cien. Si tengo cien perros, ¿cuántas colas tengo en total?

20. T: Cien.

21. E: ¿Cómo lo supiste?

22. T: Porque son cien números.

23. E: ¿Qué estás haciendo siempre, cómo lo supiste tan rápido?

24. T: Porque hay que repetir el número que tu decís.

En el extracto anterior encontré una idea que me ayudó a dar sentido a las ideas que T estaba construyendo T: "porque hay que repetir el número que tu decís" (ver línea 20). Para verificar dicha idea, le pregunté por la cantidad de colas si tenemos un millón de perros. Ante lo anterior, T respondió "dos mil trecientos cien perros" y al preguntar el porqué, señala "porque son muchos".

Luego, le pregunté a $\mathrm{T}$ sobre una cantidad indeterminada de perros, con la finalidad de que fuera capaz de expresar su regla general de otra forma. El siguiente extracto ilustra la forma en que $\mathrm{T}$ interactúa con cantidades no específicas.

25. E: $\quad$ Mira, ahora vamos a pensar en lo siguiente. No sé cuántos perros hay. Solo sé que hay muchos $^{4}$ perros. ¿Cuántas colas hay en total?

26. T: Muchas.

27. E: ¿Por qué?

28. T: Porque son muchas.

29. E: Entonces, ¿qué está pasando siempre con el número de colas?

30. T: Digo el mismo número.

31. E: $\quad Y$ si un amigo te dice: "T, en el refugio hay 6 perros, entonces hay 5 colas", ¿estarías de acuerdo con él?

${ }^{4}$ Los énfasis son míos. 
32. T: ¡No!

33. E: ¿Por qué?

34. T: Porque debe repetir el número.

Nuevamente, aparece la idea de "repetir el número" (ver líneas 16, 24 y 34).

\section{El problema de los perros y las colas (relación inversa, $x=y$ )}

Posteriormente, pregunté a T sobre la cantidad total de perros dada una cantidad de colas (relación inversa). Al igual que en la primera parte del problema, conforme fui preguntando le presenté el material manipulativo para explicar la situación y para que, potencialmente, $\mathrm{T}$ se apoyara en dicha representación al responder. Nuevamente, $\mathrm{T}$ prescindió del material manipulativo. Para los casos de 1, 2, 3, 8 y 5 colas, él brindó sus respuestas inmediatamente y su argumento fue el mismo, tal como lo muestro en el siguiente extracto.

35. E:

36. T:

37. E:

38. $\mathrm{T}$ :
Y si ahora te digo que conté 3 colas, ¿cuántos perros había en el refugio?

Tres (y lo indica con sus dedos, ver Figura 6).

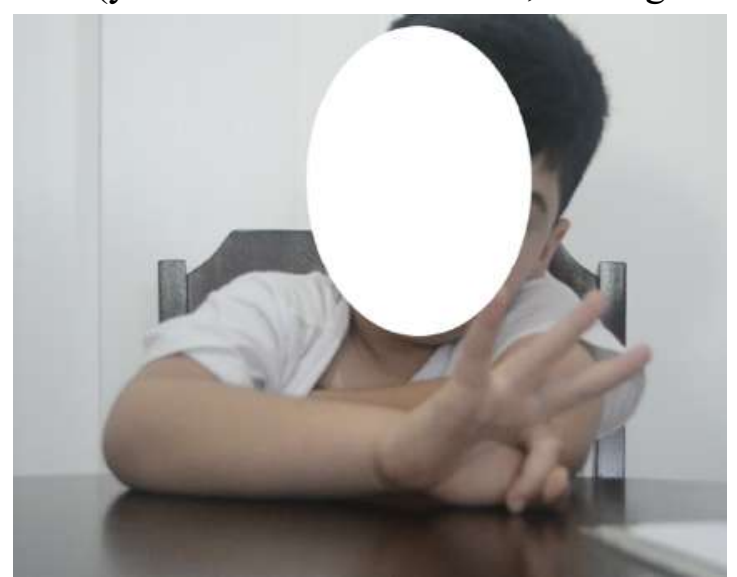

Figura 6. T representa con sus dedos la respuesta a la pregunta ¿Por qué?

Porque son tres perros.

En todas las preguntas que hice sobre cantidades específicas de colas $(1,2,3,8,5$ y 100), T respondió inmediatamente y su argumento fue siempre el mismo: "Porque son 1/2/3/8/5/100 perros". Luego, al preguntarle por la cantidad de perros cuando se cuentan un millón de colas, $\mathrm{T}$ responde "cinco, porque es mi número favorito y son los años que tengo".

Posteriormente, y dado que $\mathrm{T}$ notó $\mathrm{T}$ alguna regularidad, le pregunté por una cantidad indeterminada de colas. 
39. E: $\quad Y$ ahora por ejemplo no sé cuántas colas conté.

Solo sé que hay muchísimas colas. ¿Cuántos perros habré contado?

40. T: Muchísimos.

41. E: ¿Por qué?

42. T: $\quad$ Porque son muchísimos perros.

43. E: $\quad Y$ en este problema, cuando yo te digo un número de colas y tu me dices el número de perros, ¿qué está ocurriendo siempre?

44. T: Digo los números, el mismo que tu decís.

45. E: $\quad$ ¿Y por qué dices el mismo número que yo digo?

46. T: Porque a ti se te ocurre.

\section{El problema de los perros y los ojos (relación directa, $y=2 x$ )}

En la segunda entrevista presenté a $\mathrm{T}$ el problema de los perros y los ojos. Comencé a preguntarle por la cantidad de ojos total que hay cuando tenemos 1, 2, 3, 8 y 5 perritos. A diferencia del problema anterior, $\mathrm{T}$ se apoyó en el material manipulativo para responder y para justificar sus ideas. Específicamente, T realizó un conteo de 1 en 1 para responder a las diferentes pregunté que formulé. Por ejemplo, en la Figura 7 muestro la forma en que $\mathrm{T}$ se apoya en este material para responder la cantidad total de ojos cuando hay 2 perros (Figura 7.a), 3 perros (Figura 7.b) y 8 perros (Figura 7.c).

a.

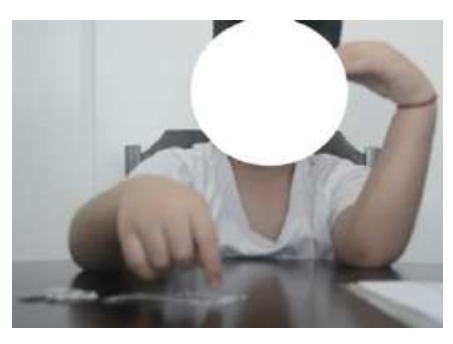

b.

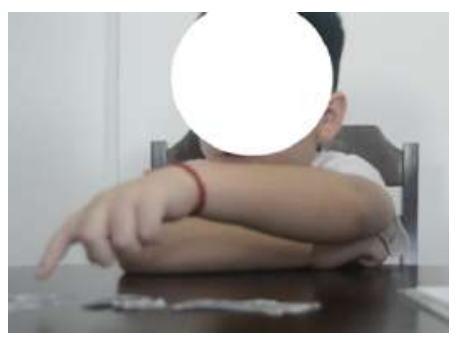

c.

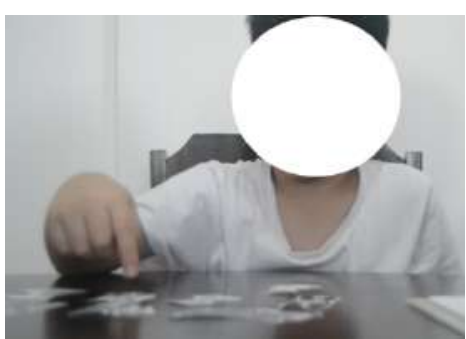

Figura 7. T cuenta la cantidad de ojos, de 1 en 1

Cuando intenté preguntar por la cantidad de ojos para cien y un millón de perros, T señaló "no sé, me demoraría muchísimo en contar".

\section{DISCUSIÓN Y CONCLUSIONES}

En este artículo describo cómo un niño de 5 años generaliza y justifica relaciones matemáticas al resolver un problema que involucra una función lineal. Si bien desde hace 
varios años la comunidad científica ha estado demostrando cómo estudiantes en escolaridad primaria generalizan relaciones matemáticas al trabajar con diferentes contenidos de carácter algebraico, los estudios centrados en estudiantes de 4-6 años son escasos. Los hallazgos expuestos aquí no buscan generalizar patrones de razonamiento algebraico de niños de 5 años, dado que la muestra informante corresponde a un niño concreto y específico. Lo que sí me parece importante de resaltar es que el interés por las justificaciones matemáticas de niños en contextos algebraicos no es algo trivial; estas favorecen que niños construyan relaciones matemáticas generales que les hagan sentido y puedan compartir con otros (ELLIS, 2007).

En este artículo busco responder a la pregunta de investigación: ¿cómo las representaciones manipulativas apoyan la construcción de la generalización y justificaciones matemáticas al resolver problemas desde un enfoque funcional al pensamiento algebraico? Mi interés por las representaciones manipulativas tiene estrecha relación con la edad del participante entrevistado, dado que la posibilidad de manipular táctilmente las representaciones podría suponer que $\mathrm{T}$ encontrara diversos tipos de relaciones y regularidades en los problemas expuestos. Sin embargo, y considerando los problemas presentados a $\mathrm{T}$, los hallazgos sugieren que este tipo de representación no constituyó una herramienta útil para generalizar y/o justificar sus ideas. Dos posibles explicaciones podrían relacionarse con esta situación: (a) quizás el material manipulativo no era rico en significados por si mismo; o (b) la experiencia escolar del participante (que se ha desarrollado manera telemática, principalmente) supone una falta de experiencias con materiales manipulativos para organizar ideas matemáticas. En línea con otras investigaciones (e.g., BLANTON; KAPUT, 2004; CASTRO; CAÑADAS; MOLINA, 2017), las representaciones manipulativas fueron útiles para realizar conteos específicos (en el problema de los perros y ojos, por ejemplo), pero no observé evidencias de que su uso apoyara la construcción de generalizaciones o justificaciones. Todo lo anterior permite indicar que ese tipo de representaciones no lleva automáticamente a niños a generalizar (CAI; MOYER, 2008). Por tanto, se hace necesario proporcionar interacciones con este tipo de material que favorezca la identificación de regularidades.

Considerando el primer problema presentado (el de los perros y colas), la forma en que emergen las generalizaciones de relaciones matemáticas es interesante; $\mathrm{T}$ fue 
percibiendo que existía una relación entre el número de perros y número de colas, y viceversa. A pesar de que existían diferentes tipos de relaciones y regularidades en el problema presentado, $\mathrm{T}$ señala una regularidad que usó de manera consistente: hay que repetir el número dado para responder (ya sea para indicar el número de colas total o número de perros), sin importar el número. Al parecer, y sin emplear o referirse al material concreto, $\mathrm{T}$ reconoce una regularidad entre la relación de la variable independiente (número de perros) y la variable dependiente (número de colas). Él indica que hay una relación coincidente entre el número de perros y el número de colas. Parece que $\mathrm{T}$ nota una regularidad, algo que siempre está pasando. Lo anterior cobra fuerza cuando $\mathrm{T}$ interactúa con cantidades indeterminadas (Mira, ahora vamos a pensar en lo siguiente. No sé cuántos perros hay. Solo sé que hay muchos perros. ¿Cuántas colas hay en total?). T logró repetir la expresión "muchos" en su respuesta, lo que consistente con sus hallazgos. Esta interacción entre T y lo indeterminado es lo que hace que la actividad que se ha desarrollado sea algebraica: las relaciones no se centran en cantidades específicas, sino que buscan abstraer las relaciones encontradas.

Durante las justificaciones de $\mathrm{T}$ al primer problema, él desarrolla argumentos que se basan en las relaciones generales encontradas (porque debe repetir el número, línea 34). Esta formar de justificar lo hace para explicar sus razonamientos, así como para convencer a otros. Reconocer si una justificación matemática es válida es un componente esencial del proceso de razonamiento matemático en general (LANNIN; ELLIS; ELLIOT, 2011), y del algebraico en particular. Por tanto, brindar oportunidades para que niños expliquen estrategias e ideas, las contrasten con otros, describan sus conjeturas y las justifiquen, entre otras, es un camino que se reconoce como importante y que favorecería generar una cultura de compartir y construir ideas dentro del aula de matemáticas.

El segundo problema presentado supuso una dificultad mayor en $\mathrm{T}$, dado que no evidenció de manera explícita algún tipo de regularidad. Lo anterior puede explicarse por el tipo de función involucrada en el problema $(y=2 x)$, dado que este tipo de situaciones supone una mayor complejidad al relacionar las variables (STEPHENS; ELLIS; BLANTON; BRIZUELA, 2017). A pesar de las dificultades encontradas por $\mathrm{T}$ al resolver este problema, la posibilidad de enfrentarlo a tareas desafiantes supone una oportunidad para afianzar su gusto por las matemáticas. 
A pesar de los énfasis en los currículos matemáticos de 3-6 años por introducir conceptos e ideas algebraicas (e.g., SUBSECRETARÍA DE EDUCACIÓN PARVULARIA, 2018) y fomentar la argumentación y justificación matemática en general (CORNEJO-MORALES; ALSINA, 2020), se hace necesario proporcionar evidencias que muestren cómo es posible llevar esto a cabo en las aulas. Con este estudio brindo orientaciones de cómo es posible desarrollar un tipo de pensamiento que favorece que niños construyan relaciones matemáticas más abstractas y borrar ideas de que el álgebra va más allá que el uso de letras y símbolos.

\section{REFERENCIAS}

AMIT, M.; NERIA, D. "Rising to the challenge": using generalization in pattern problems to unearth the algebraic skills of talented pre-algebra students. ZDM, Dordrecht, v. 40, n. 1, p. 111-129, 2008.

BAROODY, , A. J. How and when should place-value concepts and skills be taught? Journal for Research in Mathematics Education, v. 21, n 4, p. 281-286, 1990.

BAROODY, A. The developmental bases for early childhood number and operations standards. In: CLEMENTS, D. H.; SARAMA, J. (Eds.), Engaging young children in mathematics: standards for early childhood mathematics. Mahwah, NJ, Lawrence Erlbaum Associates. 2004. p. 173-219.

BASTABLE, V.; SCHIFTER, D.. Classroom stories: Examples of elementary students engaged in early algebra. In: KAPUT, J. J.; BLANTON, M. L.; CARRAHER, D. W. (Eds.), Algebra in the early grades. Mahwah, NJ, Lawrence Erlbaum Associates. 2008. p. 165-184.

BLANTON, M. L.; OTÁLORA, Y.; BRIZUELA, B.; GARDINER, A.; SAWREY, K.; GIBBINS, A.; KIM, Y. Exploring Kindergarten Students' Early Understandings of the Equal Sign. Mathematical Thinking and Learning, v. 20, n. 3, p. 167-201, 2018.

BLANTON, M. L. Algebra and the elementary classroom. Transforming thinking, transforming practice. Portsmouth, NH. 2008.

BLANTON, M. L. Algebraic reasoning in grades 3-5. In: BATTISTA, M. T. (Ed.), Reasoning and sense making in the mathematics classroom. Grades 3-5. Reston, VA, NCTM, 2017. p. 67-102.

BLANTON, M. L., BRIZUELA, B., STEPHENS, A., KNUTH, E., ISLER, I., GARDINER, A., STROUD, R., FONGER, N., STYLIANOU. Implementing a Framework for Early Algebra. In: KIERA, C. (Ed.), Teaching and Learning Algebraic Thinking with 5-to-12-Year-Olds, Dordrecht. 2018. p. 27-49.

BLANTON, Maria L. e KAPUT, James J. Elementary grade students' capacity for functional thinking. 2004, [S.1: s.n.], 2004. p. 135-142. 
BLANTON, Maria L. e KAPUT, James J. Generalizing and progressively formalizing in a third-grade mathematics classroom: Conversations about even and odd numbers. 2000, Columbus, OH: ERIC Clearinghouse for Science, Mathematics, and Environmental Education., 2000. p. 115-119.

BOALER, J.; HUMPHREYS, C. Connecting mathematical ideas. Middle school video cases to support teaching and learning. Portsmouth, NH, Heinemann, 2005.

CAI, J.; MOYER, J. C. Developing Algebraic Thinking in Earlier Grades : Some Insights from International Comparative Studies. In: GREENES, C. E.; RUBENSTEIN, R. (Eds.), Algebra and algebraic thinking in school mathematics. Reston, VA, NCTM, 2008. p. 169-192.

CAÑADAS, M. C.; CASTRO, E. A proposal of categorisation for analysing inductive reasoning. PNA, Granada, v. 1, n. 2, p. 67-78, 2007.

CAÑADAS, M. C.; MOLINA, M. Una aproximación al marco conceptual y principales antecedentes del pensamiento funcional en primeras edades. In CASTRO, E. (Ed.), Investigación en Educación Matemática. Homenaje a Luis Rico. Granada, España: Comares, 2016. p. 209-218.

CARRAHER, D. W.; SCHLIEMANN, A. D. Algebraic reasoning in elementary school classrooms. In: LAMBDIN, D.; LESTER, F. (Eds.), Teaching and learning mathematics: Traslating research to the classroom. Reston, VA, NCTM, 2010. p. 2329.

CARRAHER, D. W.; SCHLIEMANN, A. D. Powerful ideas in elementary school mathematics. In: ENGLISH, L.; KIRSHNER, D (Eds.), Handbook of International Research in Mathematics Education: Third Edition, n. 2, p. 191-218, 2015.

CARRAHER, D. W.; SCHLIEMANN, A. D. Early algebra and algebraic generalization. Handbook of research on mathematics education, p. 669-705, 2007.

CASTRO, E.; CAÑADAS, M. C.; MOLINA, M. Pensamiento funcional mostrado por estudiantes de Educación Infantil. Edma, Madrid, v. 6, n. 2, p. 1-13, 2017.

CASTRO, E.; CASTRO, E. Enseñanza y aprendizaje de las matemáticas en educación infantil. Granada, España, Pirámide, 2016.

COHEN, L.; MANION, L.; MORRISON, K. Research methods in education (Octava edición). Nueva York, NY, Routledge, 2018.

CORNEJO-MORALES, C.; ALSINA, Á. La argumentación en los currículos de educación matemática infantil. Edma 0-6: Eduación Matemática en la Infancia, Madrid, v. 9, n. 1, p. 12-30, 2020.

ELLIS, A. B. A taxonomy for categorizing generalizations: Generalizing actions and reflection generalizations. Journal of the Learning Sciences, v. 16, n. 2, p. 221-262, 2007.

FUSON, K. C. Pre-K to grade 2 goals and standards: Achieving 21st century mastery for all. In: CLEMENTS, D. H.; SARAMA, J. (Eds.), Engaging Young Children in Mathematics: Standards for Early Childhood Mathematics. Mahwah, NJ, Erlbaum, 
2004. p. 105-148.

GINSBURG, H. Entering the child's mind: The clinical interview in psychological research and practice. Nueva York, NY, Cambridge University Press, 1997.

HERNÁNDEZ, R.; FERNÁNDEZ, C.; BAPTISTA, M. Metodología de la investigación (Quinta edición). México, D.F., McGraw-Hill, 2020.

LANNIN, J.; ELLIS, A. B.; ELLIOTT, R. Developing essential understanding of mathematical reasoning. Pre-K - Grade 8. Reston, VA, NCTM, 2011.

LESH, R. A.; BEHR, M; POST, T. Rational numbers relations and proportions. In: C. JANVIER (Ed.), Problems of representation in the teaching and learning of mathematics Hillsdale, NJ, Lawrence Erlbaum Associates. 1987. p. 41-58.

MASON, J. Expressing generality and roots of algebra. In: BERNARZ, N.; KIERAN, C.; LEE, L. (Eds.), Approaches to Algebra: Perspectives for Research and Teaching. Dordrecht, Springer Netherlands, 1996. p. 65-86.

MORALES, R.; CAÑADAS. M. C.; BRIZUELA, B.; GÓMEZ, P. Relaciones funcionales y estrategias de alumnos de primero de Educación Primaria en un contexto funcional. Enseñanza de las Ciencias, Barcelona, v. 36, n. 3, p. 59-78, 2018.

PIAGET, J.; INHELDER, B. The child's conception of space. Nueva York, NY, Routledge, 1967.

PINTO, E. Generalización de estudiantes de $3^{\circ}$ a $6^{0}$ de Educación Primaria en un contexto funcional del álgebr escolar. 2019. 341 f. Universidad de Granada, 2019.

RAMIREZ, R.; BRIZUELA, B.; BLANTON, M. L. Kindergarten and First-Grade Students' Understandings and Representations of Arithmetic Properties. Early Childhood Education Journal, v. first online, 2020.

RUSSELL, S.; SCHIFTER, D., BASTABLE, V. Connecting arithmetic to algebra. Portsmouth, NH, Heinamnn, 2011.

SARAMA, J.; CLEMENTS, D. H. Early childhood mathematics education research. Learning trajectories for young children. Nueva York, NY, Routledge, 2009.

SMITH, E. Stasis and change: Integrating patterns, functions, and algebra throughout the K-12 curriculum. In: KILPATRICK, J.; MARTIN, W. G.; SCHIFTER, D. (Eds.), A research companion to Principles and Standards for School Mathematics. Reston, VA, NCTM, 2003. p. 136-150.

STEPHENS, A.; ELLIS, A. B.; BLANTON, M. L.; BRIZUELA, B. Algebraic thinking in the elementary and middle grades. CAI, J. (Ed.), Compendium for research in Mathematics Education. Reston, VA, NCTM, 2017. p. 386-420.

SUBSECRETARÍA DE EDUCACIÓN PARVULARIA. Bases Curriculares Educación Parvularia. Santiago, Chile, Ministerio de Educación, 2018.

TORRES, M. D., CAÑADAS, M. C.; MORENO, A.; GÓMEZ. P. Estructuras en las formas directa e inversa de una función por estudiantes de 7-8 años. Uniciencia, San José, v. 35, n. 2, p. 1-19, 2021. 
WARREN, Elizabeth. Young children's ability to generalise the pattern rule for growing patterns. Proceedings of the 29th Conference of the International group for the Psychology of Mathematics Education, v. 4, p. 305-312, 2005.

Submetido em 30 de março de 2021. Aprovado em 03 de maio de 2021. 